\title{
单星条件下基于被动测距的弹道参数估计性能分析
}

\author{
李 淼, 黄 源, 孙 扬, 林再平 \\ (国防科技大学 电子科学学院, 湖南 长沙 410073)
}

\begin{abstract}
摘要: 在天基光学监视系统中, 根据有限的导弹助推段观测信息估算目标的弹道参数是衡量系统性 能的重要指标, 对支持目标识别分类、判定威胁程度都有重要意义。本文针对单星观测条件下提高 弹道参数估计精度的迫切需求, 提出了天基条件下利用氧气 $\mathrm{A}$ 吸收带被动测距提高估计精度的方法。 利用 MODTRAN 对不同大气模型、不同气象、不同信噪比条件下的天基被动测距能力进行了仿真分 析, 在此基础上深入分析被动测距技术对弹道参数估计精度的提升能力, 并与传统经验方法进行对 比。仿真表明本文方法能够有效提高单星观测下的弹道参数估计精度。
\end{abstract}

关键词: 天基光学监视系统; 助推段; 弹道参数估计; 被动测距

中图分类号：TP183 文献标识码：A 文章编号：1001-8891(2020)04-0328-07

\section{Performance Analysis of Ballistic Estimation Based on Passive Ranging Using Single Satellite}

\author{
LI Miao, HUANG Yuan, SUN Yang, LIN Zaiping \\ (College of Electronic Science and Technology, National University of Defense Technology, Changsha 410073, China)
}

\begin{abstract}
For space-based optical surveillance systems, estimated ballistic parameters are important output specifications based on limited boost-phase measures. They are useful for target classification and threat assessment. To improve estimation accuracy under a single satellite, passive ranging based on the atmospheric absorption of oxygen " $\mathrm{A}$ " band is introduced in this study. The performance of passive ranging under different atmosphere models, weather scenes, and signal-to-noise ratio are discussed and simulated separately in moderate resolution atmospheric transmission. Furthermore, the performance of ballistic estimation based on passive ranging is compared with an experiential method. Simulation results show that the proposed method can effectively improve the accuracy of ballistic estimation.
\end{abstract}

Key words: space-based optical surveillance system, boost-phase, ballistic estimation, passive ranging

\section{0 引言}

在天基光学监视系统中, 根据有限的单星观测数 据实现目标识别、威胁评估等工作是系统面临的常见 问题。但简单观测和复杂信息提取之间存在明显矛 盾, 是亟需攻关的热点和难点。当今, 常见方法是在 单星条件下利用获得的目标助推段信息, 估计目标弹 道参数 (包括发射位置、射程等), 与已建立的弹道 模板数据库进行匹配, 进而实现目标识别等处理。针 对此需要, 美军已建立了一个包含约 7200 条数据的 导弹数据库 ${ }^{[1]}$ 。从这一过程可看出, 准确估计目标弹 道参数是提升系统整体性能的重要途径。传统方法主
要是在粗略经验和弹道模型下进行弹道参数估计, 但 经验数值误差较大, 所得估计结果精度较低。针对此 问题, 本文将被动测距引入单星天基观测, 并对其性 能进行分析。

在被动测距中领域, 基于红外辐射传输特性的 单站被动测距技术受到了众多研究者的关注, 并在 近年发展迅速。当前主要的单站被动测距方法有 ${ }^{[2]}$ : (1)基于辐射强度变化特性的单站被动测距方法, 该 方法假设目标为辐射强度恒定的点源, 当作匀速直 线运动时, 利用光谱辐射强度与距离的映射关系估 算距离 ${ }^{[3]}$; (2) 基于目标红外成像几何特征的单站被动 测距方法, 该方法利用目标尺度大小与距离的关系 
估计目标距离 ${ }^{[4]}$; (3)单站红外双波段被动测距方法, 该方法根据红外辐射在不同波段衰减不同实现被动 测距 ${ }^{[5]}$; (4)基于氧气 $\mathrm{A}$ 吸收带光谱特性的被动测距方 法, 该方法根据氧气 A 吸收带吸收率与距离的关系实 现被动测距 ${ }^{[6]}$ 。

基于上述 4 种方法分别有如下优缺点: 方法(1)适 用条件要求苛刻, 辐射恒定和匀速直线运动等假设条 件难以在实际应用中满足。方法(2)虽可适用非线性目 标, 但需建立目标形态与距离对应关系的先验信息, 难以应用到非合作目标。方法(3)具有实时性好、适用 非线性目标的优点, 但选择的两个红外波段吸收率受 $\mathrm{CO}_{2}$ 、水等多种成分影响, 易受气象因素的影响, 稳 定性差。方法(4)自 2005 年被提出, 受到了美国空军 技术研究所的重视, 对光谱特征和大气传输特性展开 了深入研究, 证明了在氧气 A 吸收带不受其他气体成 分的影响, 具有实时测距、不受气象因素影响、适用 所有运动形式等优点。在 2010 年分别对 F16 飞机和 猎鹰 9 运载火箭进行了测距。在 $90 \mathrm{~s}$ 的实验中, 最大 探测距离约为 $90 \mathrm{~km}$, 最大误差小于 $5 \%$, 平均误差 小于 $3 \%{ }^{[7]}$ 。基于上述分析, 本文结合天基观测条件, 主要对基于氧气吸收带的被动测距方法进行研究。

\section{1 天基条件下的单站被动测距方法}

自 20 世纪 60 年代, 处于 $758 \sim 778 \mathrm{~nm}$ 的氧气 A 吸收带一直受到大气遥感领域研究者的关注。特别是 随着精细光谱测量技术的快速发展, 氧气 $\mathrm{A}$ 吸收带受 到越来越多的重视 ${ }^{[8]}$ 。地球大气中的氧气所占比例大 约为 $20.947 \%$, 且浓度相对稳定。氧气 A 吸收带是因 氧分子磁偶极矩转动跃迁而形成的, 不受其他气体吸 收的干扰, 氧气为唯一的吸收气体, 被认为是被动测 距的潜力谱段。

在天基观测条件下, 目标辐射信号经过大气传输 后到达探测传感器。由于大气传输中存在的吸收、散 射及大气湍流等因素的影响, 只有原信号中的部分能 量能够被传感器接受 ${ }^{[9]}$, 如图 1 所示。

辐射信号经过一定厚度的均匀吸收介质传播后, 一部分能量被介质吸收, 透过光的强度减弱 ${ }^{[10]}$ 。在传 播过程中吸收介质厚度越大、浓度越强, 衰减越明显。 这一过程可表述为式(1):

$$
-\frac{\mathrm{d} I}{I}=\alpha \mathrm{d} x
$$

式中: $\mathrm{d} x$ 代表在均匀介质中的传输路程; 原辐射光强 为 $I$; 衰减辐射光强为 $\mathrm{d} I ; \alpha$ 代表介质吸收系数。一
般认为 $\alpha$ 是波长的函数 $\alpha(\lambda)$ 。从式(1)可看出, 衰减程 度与光谱的吸收特性和路径长度有关。

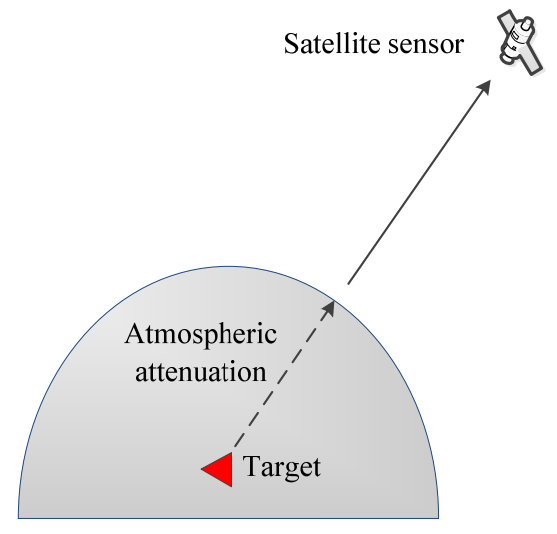

图 1 天基被动测距示意图

Fig.1 Passive ranging for space-based observation

将式(1)从 $0 \sim x$ 积分, 可得经过 $x$ 距离传输后的 光辐射光强:

$$
I(x)=I(0) \mathrm{e}^{-\alpha(\lambda) x}
$$

式中: $I(0)$ 代表在 $x=0$ 处的原始光强。

则吸收率可通过式(3)计算得:

$$
\begin{aligned}
A & =\frac{I(0)-I(x)}{I(0)} \\
& =1-\mathrm{e}^{-\alpha(\lambda) x}
\end{aligned}
$$

在实际情况中, 在氧气 A 吸收带, 传输衰减不仅 包含氧气分子吸收, 还包含气溶胶散射等, 同时任何 测量都会受到测量系统本身的限制, 只能测得一定带 宽和光谱分辨率的光谱 ${ }^{[11]}$ 。因此实际测量到的目标辐 射光谱应写为:

$$
I_{\mathrm{m}}=I_{\mathrm{o}} \cdot T_{\text {scat }} \cdot T_{\mathrm{O}_{2}} \cdot R_{\text {sensor }}
$$

式中: $I_{\mathrm{o}}$ 为原始光谱强度; $T_{\text {scat }} 、 T_{\mathrm{O}_{2}}$ 和 $R_{\mathrm{sensor}}$ 分别代 表与气溶胶散射有关的透过率、氧气吸收导致的透过 率、与传感器的响应函数。

在探测过程中, 直接获得的是 $I_{\mathrm{m}}$, 无法精确获得 $I_{0} 、 T_{\text {scat }}$ 和 $R_{\text {sensor }}$ 的独立值。但 $T_{\text {scat }}$ 和 $R_{\text {sensor }}$ 缓慢变化, 可视为观测值中的低频部分; 相对而言, 与氧气分子 吸收相关的 $T_{\mathrm{O}_{2}}$ 可视为高频部分。基于此特点, 可通 过基线估计的方法剔除 $T_{\text {scat }}$ 和 $R_{\text {sensor }}$ 的影响 ${ }^{[6]}$, 即:

$$
I_{\mathrm{b}}=I_{\mathrm{o}} \cdot T_{\text {scat }} \cdot R_{\text {sensor }}
$$

式中: $I_{\mathrm{b}}$ 为建立的基线。对 $I_{\mathrm{b}}$ 的估计称为基线拟合过 程, 即为利用得到的光谱拟合出吸收前的光谱。在 $\mathrm{A}$ 
带两翼存在氧气吸收作用薄弱近似可忽略的两个频 带, 相对于氧气吸收带称其为非谐振频带, 波数分别 为 $13200 \sim 13360 \mathrm{~cm}^{-1}$ 和 $14590 \sim 19900 \mathrm{~cm}^{-1}$ 。研究这 两个非谐振频带的光谱特性, 利用插值拟合可得吸收 波段的基线 ${ }^{[9]}$, 如图 2 所示。

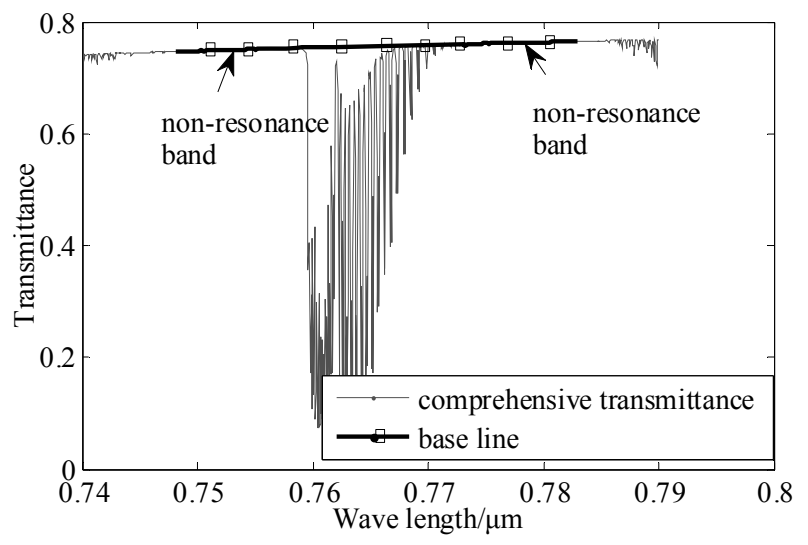

图 2 基线拟合示意图

Fig.2 The sketch diagram of baseline fitting 将拟合的基线带入式(1)可得：

$$
I_{\mathrm{m}}=I_{\mathrm{b}} \cdot T_{\mathrm{O}_{2}}
$$

即可计算得氧气透过率为:

$$
T_{\mathrm{O}_{2}}=I_{\mathrm{m}} / I_{\mathrm{b}}
$$

从式(7)可看出, 准确的估计基线是计算氧气透过 率的关键。

根据基线拟合原理, 氧气 $\mathrm{A}$ 吸收带两翼的非谐振 频带氧气分子吸收作用几乎为零, 即:

$$
T_{\mathrm{O}_{2}} \approx 1
$$

因此, 可通过插值的方法拟合 $I_{\mathrm{b}}$, 即:

$$
I_{\mathrm{b}} \approx I_{\mathrm{m}(\text { 带外 })}
$$

从而氧气吸收率可表示为:

$$
A=1-T_{\mathrm{O}_{2}}=1-\frac{I_{\mathrm{m}}}{I_{\mathrm{b}}}
$$

进而, 目标距离可通过式(11)计算得:

$$
x=\frac{1}{\alpha(\lambda)} \ln \frac{I_{\mathrm{b}}}{I(x)}
$$

综上所述, 在均匀介质中, 通过实时测量 3 个谱 段的辐射强度, 即可建立氧气吸收率 (或大气透过率) 与目标距离之间的关系。由于通过基线估计消除了气 溶胶散射和气象因素的影响, 在理论上证明了该方法 不易受气象因素的影响, 具有较强的稳健性。

上述分析都是基于均匀传输介质展开的, 而实际
地球大气的浓度是随着高度不断变化的。为了更加完 整地分析在天基观测条件下基于氧气 $\mathrm{A}$ 吸收带被动 测距方法的有效性和稳健性，下文利用 MODTRAN (MODerate resolution atmospheric TRANsmission) 软 件进行仿真。

\section{2 单星测距性能仿真分析}

MODTRAN 是当前国际上较为成熟的大气传输 仿真软件, 是基于大量大气实验提供的丰富数据建立 的, 大气透过率的计算精度较高。本文基于此软件仿 真氧气 $\mathrm{A}$ 带大气透过率与目标高度的关系。

\section{1 不同大气模型的影响}

随着纬度和季节的变化, 地球大气随之上升或下 降, 大气中的氧气浓度也随之发生变化。将这种随着 纬度和季节变化而发生的地球大气自身变化统称为 大气模型变化。为了展示不同大气模型对氧气 $\mathrm{A}$ 吸收 带被动测距方法的影响, 仿真了 6 种大气模型下目标 高度与氧气吸收率的关系。这 6 种大气模型分别为: 美国标准大气 (US standard)、热带大气（tropical）、 中纬度夏季大气 (midlatitude summer)、中纬度冬季 大气 (midlatitude winter)、近北极夏季大气 ( subarctic summer)、近北极冬季大气 (subarctic winter)。仿真 气象条件为无云, 天顶角为 $180^{\circ}$ (星下点)。仿真结 果如图 3 所示。

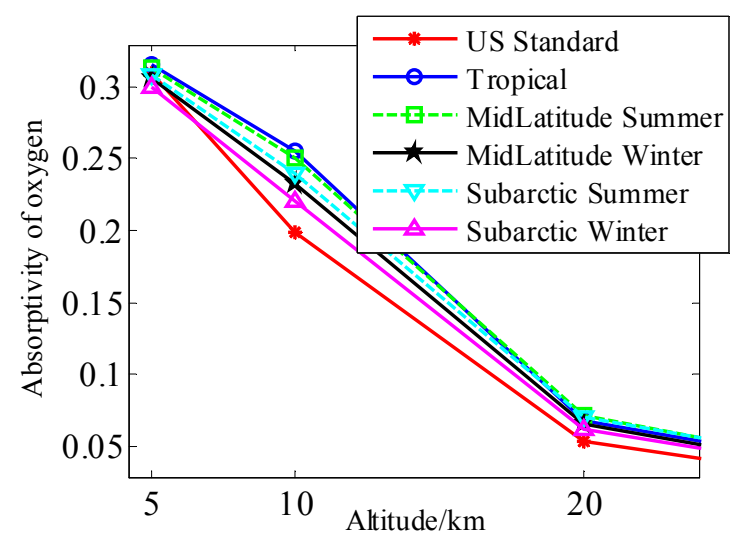

图 3 大气模型对被动测距的影响

Fig.3 The effect of atmospheric model for passive ranging

从图 3 中可看出, 在不同纬度和季节下, 目标高 度与氧气吸收率的关系略有差异, 目标高度较低时这 种差异较为明显。大气模型是不同温度变化、水汽分 布等多重因素综合作用的概括。因此，一天内不同温 度变化也可能对测距结果产生影响。在实际应用中, 为了确保足够的测距精度, 应利用探空气球等手段, 
获取当地、当时的准确大气模型, 为估算目标距离提 供基本输入。考虑到大气模型等的缓变性, 大气数据 采样间隔可根据实际情况设定。

\section{2 不同气象条件的影响}

下文对气象条件对氧气 A 吸收带测距方法的影 响进行仿真分析。考虑的气象条件包括: 气溶胶影响、 雾影响、降雨影响。仿真是在美国标准大气模型下进 行的, 天顶角 $180^{\circ}$ 。

气溶胶散射是大气散射作用的主要类型之一, 发 生在波长与散射粒子大小差不多时。主要的散射粒子 包括: 烟雾、尘埃、靁等。下文对不同气溶胶条件下 的被动测距性能进行仿真分析。仿真中两种气溶胶模 型为视距 $23 \mathrm{~km}$ 乡村气溶胶和视距 $5 \mathrm{~km}$ 乡村气溶胶。 仿真结果如图 4 所示。

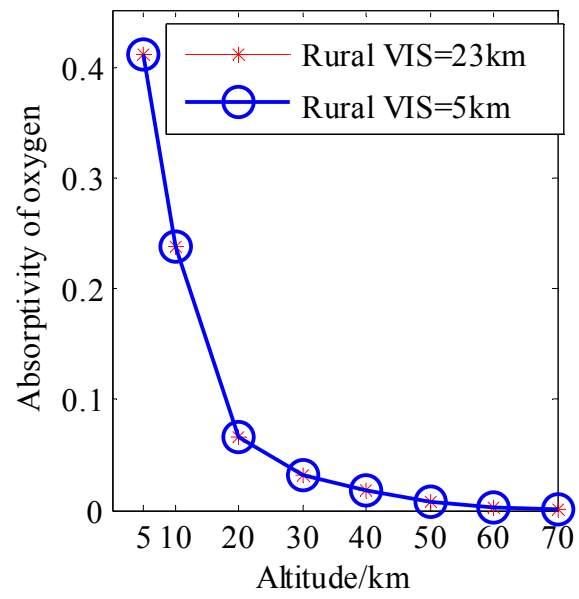

图 4 气溶胶对被动测距的影响

Fig.4 The effect of aerosol for passive ranging

当空气中水汽充足且相对湿度达到一定条件时, 水汽便会凝结为细微小水滴悬浮于空中, 使得能见度 降低, 这种天气现象称为雾。辐射雾 (fog radiation) 和平流雾 (fog advection) 是雾的两种主要形式。辐 射雾是由于夜间地面辐射冷却, 使得空气中的水汽达 到饱和导致的。平流雾是暖湿空气移动到较冷的陆地 或水面时, 因下部冷却而形成的雾, 一般出现在海边。 下面对两种雾的影响进行仿真分析, 仿真结果如图 5 所示。

当空气中的水滴达到一定程度时, 将会降落到地 面形成降雨, 按照降雨量的不同可分为小雨、中雨和 大雨。下文对不同降雨量下氧气吸收率与高度的关系 进行仿真, 仿真结果如图 6 所示。

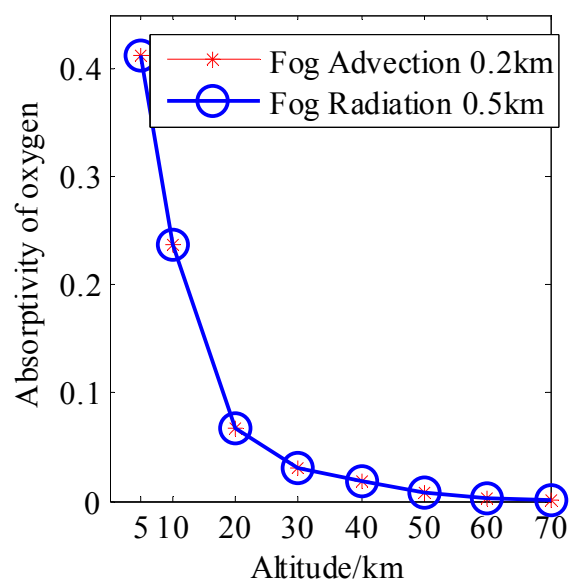

图 5 雾对被动测距的影响

Fig.5 The effect of fog for passive ranging

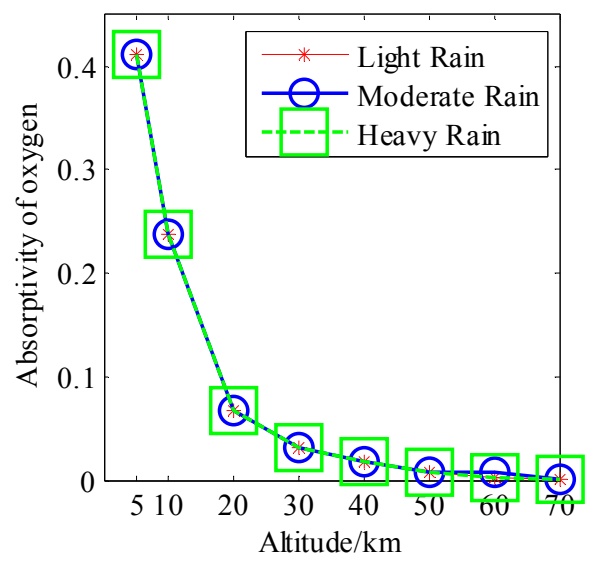

图 6 雨对被动测距的影响

Fig.6 The effect of rain for passive ranging

从上述在不同气溶胶、雾、雨条件下的仿真结果 表明, 气象条件对天基观测下的被动测距性能影响较 小。分析其原因，一方面是由于通过基线拟合消除了 气象条件对氧气 A 带吸收率的影响, 另一方面与气象 变化主要发生在大气底层有关。上述仿真证明了基于 氧气 A 吸收带被动测距方法的稳健性。

\section{3 不同传感器信噪比的影响}

本小节对不同传感器信噪比下被动测距性能进 行仿真。仿真大气模型为中纬度冬季模型, 天顶角为 $180^{\circ}$, 目标高度分别为 $13 \mathrm{~km} 、 15 \mathrm{~km}$ 和 $17 \mathrm{~km}$ 。每种 工况下进行蒙特卡罗仿真, 信噪比主要考虑仪器本身 的热噪声。仿真结果如图 7 所示。 


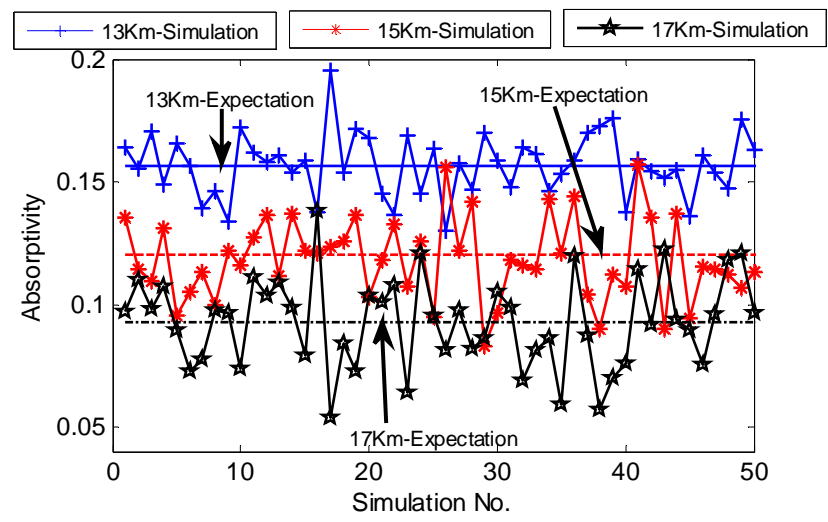

(a) $\mathrm{SNR}=50$

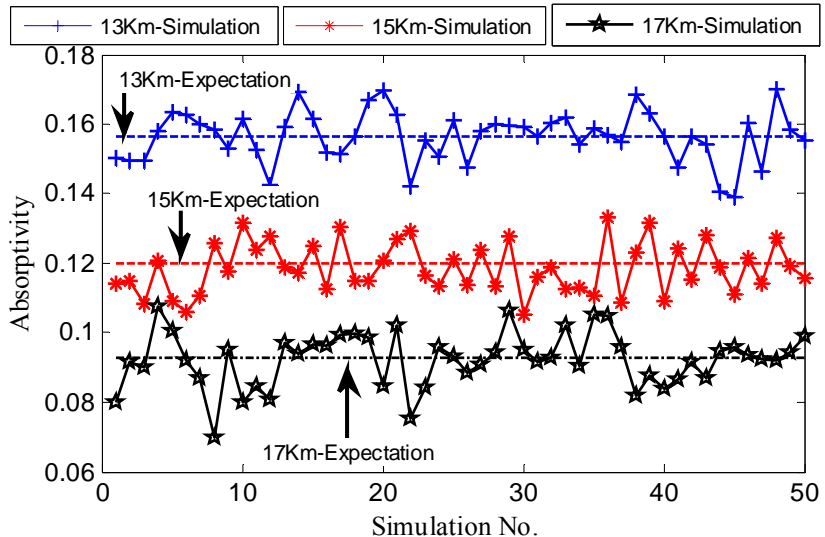

(b) $\mathrm{SNR}=100$

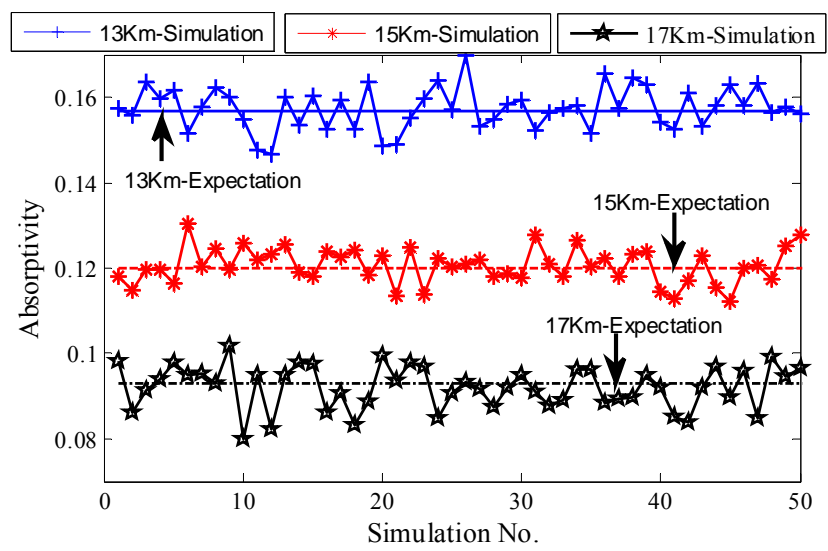

(c) $\mathrm{SNR}=150$

图 7 不同信噪比对被动测距的影响

Fig.7 The effect of SNR for passive ranging

从上述仿真可看出, 在 SNR 为 50 条件下, $15 \mathrm{~km}$ 与 $17 \mathrm{~km}$ 的分辨正确率大约为 $75 \%, 15 \mathrm{~km}$ 与 $13 \mathrm{~km}$ 的分辨正确率约为 $45 \%, 13 \mathrm{~km}$ 与 $17 \mathrm{~km}$ 的分辨正确 率约为 $90 \%$, 难以实现高可靠测距; 在 SNR 为 100 条件下, $15 \mathrm{~km}$ 与 $17 \mathrm{~km}$ 的分辨正确率大约为 $100 \%$, $15 \mathrm{~km}$ 与 $13 \mathrm{~km}$ 的分辨正确率约为 $93 \%, 13 \mathrm{~km}$ 与 $17 \mathrm{~km}$ 的分辨正确率约为 $100 \%$, 整体距离分辨性能较好; 在 SNR 为 150 条件下， $13 \mathrm{~km} 、 15 \mathrm{~km} 、 17 \mathrm{~km}$ 相互间
的分辨正确率都可达 $100 \%$ ，此情况距离分辨性能最 佳, 但对传感器性能要求也最苛刻, 实现难度较大。 综合分析得出, 传感器信噪比 $\geqslant 100$ 时, 能较好 地区分 $13 \mathrm{~km}$ 与 $15 \mathrm{~km} 、 15 \mathrm{~km}$ 与 $17 \mathrm{~km}$ 目标位置, 可认为在此条件下测距精度约为 $2 \mathrm{~km}$, 综合可信度可 达 $97 \%$, 满足一般工程需要。通过引入被动测距信息, 这将大大提高单星条件下的目标参数估计性能, 后续 仿真将在此基础上进行。

\section{3 基于单星测距的弹道参数估计性能分析}

目标上升到一定高度后, 第一次被天基监视系统 捕获, 将该点称为首次观测点。传统方法中, 根据从 首次观测到末次观测有限的视线观测、经验高度数据 及其他约束, 可大致拟合出目标弹道, 并反向推算出 目标发射点位置。单星观测条件下的弹道参数估计如 图 8 所示。首次观测和末次观测是确定弹道平面的主 要依据, 结合首次观测和末次观测的经验高度可估算 末次观测位置、速度、倾角等参数。根据粗圆弹道理 论即可估算导弹最终的射向、射程和落点。

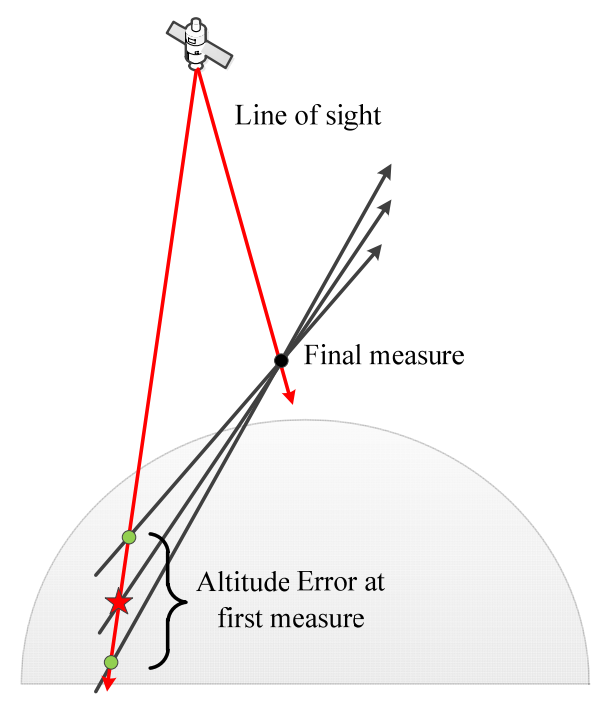

图 8 弹道参数估计示意图

Fig.8 The processing of ballistic estimation

从上述描述可看出, 单星条件下仅二维观测信 息, 传统方法主要通过经验值实现粗略三维定位。本 文通过光学被动测距, 引入测距信息, 使得测量信息 更加丰富, 从而可提高整体弹道参数估计精度。由于 随着目标高度增加, 大气衰减作用逐渐减弱, 氧气吸 收带与目标高度的线性关系逐渐模糊, 因此本文重点 分析首次观测点被动测距对弹道参数估计精度的影 响。具体仿真分析结果如下文所述。

在本仿真中, 取首次观测点高度经验值为 $15 \mathrm{~km}$, 
根据工程经验, 在无被动测距条件下先验高度误差范 围一般为 $[-10 \mathrm{~km}, 10 \mathrm{~km}]$, 对于被动测距条件下高度 误差根据 2.3 节仿真结果取值为 $[-2 \mathrm{~km}, 2 \mathrm{~km}]$ 。

\section{1 发射点估计误差分析}

下文通过理论分析分别就无被动测距和有被动 测距两种情况下的发射点估计误差进行对比, 理论分 析对比结果如图 9 和图 10 所示。

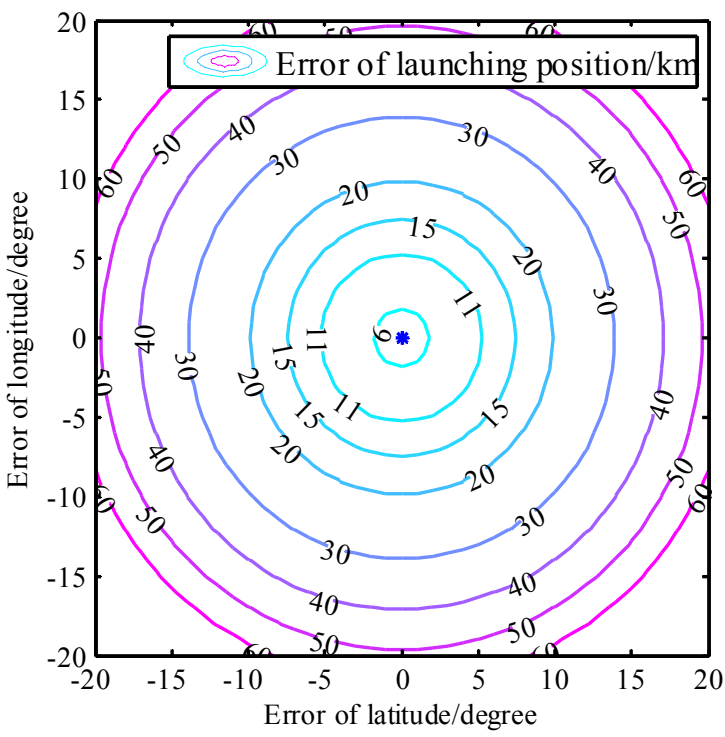

图 9 基于传统经验值的发射点估计误差

Fig.9 The error of launching position with engineering experience

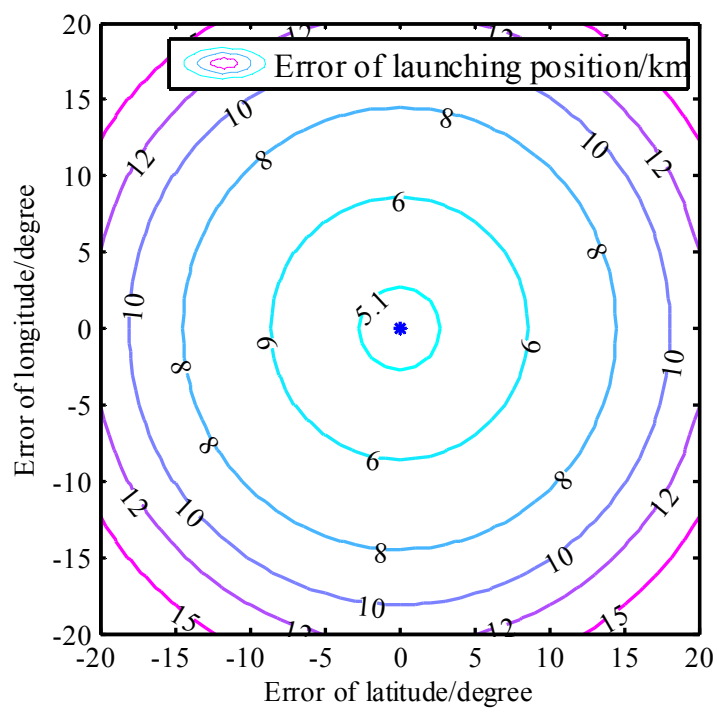

图 10 利用被动测距条件下发射点估计误差

Fig.10 The error of launching position with passive ranging

从图 9 和图 10 可看出, 在单星条件下, 通过被 动测距对首次观测点的定位, 可明显提高发射点估计 精度。在偏离星下点 $20^{\circ}$ 的视角下, 当采用传统经验 值发射点估计误差可达 $50 \mathrm{~km}$; 当采用被动测距时发
射点估计误差约为 $12 \mathrm{~km}$ 。随着视线角度的减小, 发 射点估计误差逐步减小, 在星下点的误差主要是由水 平运动距离导致的。

\section{2 射向、射程、落点误差分析}

下文通过具体目标仿真对射向、射程和落点误差 性能进行分析。3 个弹道目标分别如图 11 所示, 其中 目标 1 射程约为 $1500 \mathrm{~km}$, 目标 2 射程约为 $4000 \mathrm{~km}$, 目标 3 射程约为 $10000 \mathrm{~km}$ 。假设首次观测高度约为 $15 \mathrm{~km}$, 末次观测高度约为 $160 \mathrm{~km}$ 。

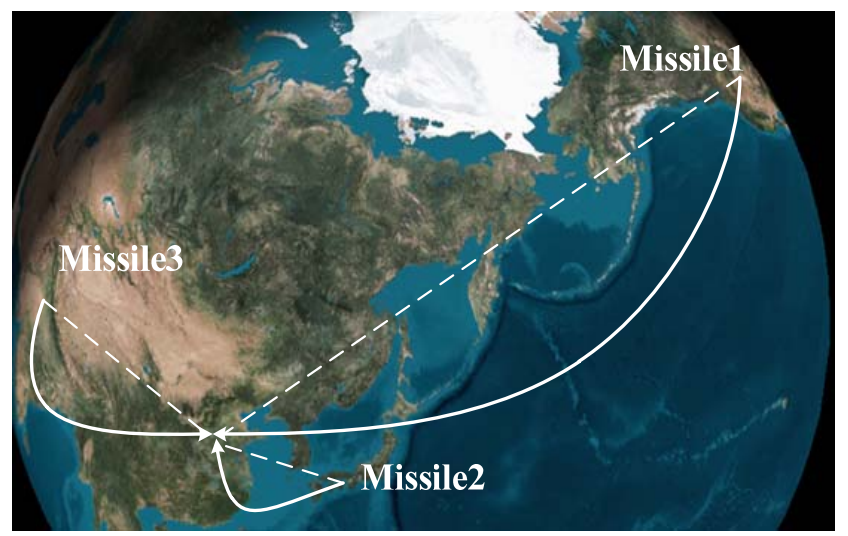

图 11 弹道估计仿真场景

Fig.11 The simulation scene for ballistic estimation

本文被动测距条件下和未使用被动测距的传统 经验方法条件下, 射向、射程和落点的估计误差展示 如图 12 14。

从图 12 14 可看出, 采用被动测距后, 初始观 测点高度定位误差减小, 从而导致弹道目标的射向、 射程和落点估计精度都有明显提高。上述仿真结果证 明了被动测距在天基监视系统中的重要作用。

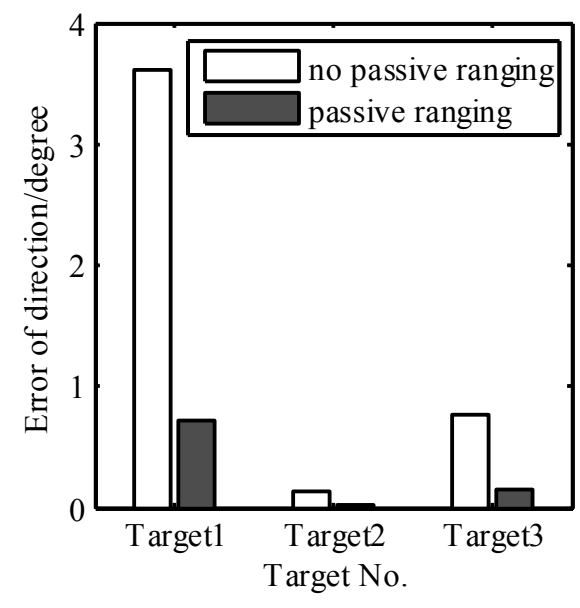

图 12 射向误差比较

Fig.12 The comparison of error of direction 




图 13 射程误差比较

Fig.13 The comparison of error of range

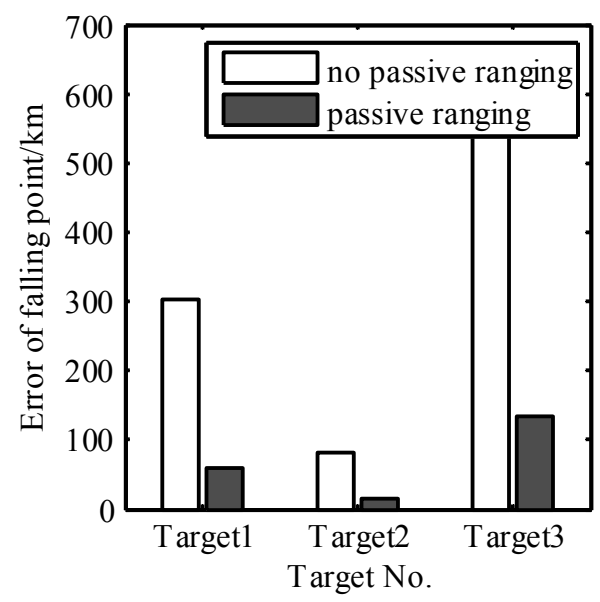

图 14 落点误差比较

Fig.14 The comparison of error of falling point

\section{4 结论}

本文通过对比当前主要的单站被动测距技术, 将 基于氧气 $\mathrm{A}$ 吸收带的被动测距方法引入天基光学监 视系统中的单星弹道参数估计。详细介绍了氧气 $\mathrm{A}$ 吸 收带的光谱特性和被动测距的基本原理, 通过 MODTRAN 软件对不同大气条件、不同气象条件、 不同传感器信噪比条件下的被动测距进行仿真分析。 通过理论分析和具体实例, 分析了首次观测点被动测 距对目标发射点、射向、射程、落点等弹道参数的精 度提升作用。

\section{参考文献：}

[1] 黄源. 弹道模板的构建与匹配技术研究[D]. 长沙: 国防科技大学, 2015.

HUANG Yuan. Research of Establishing And Matching of Ballistic Template[D]. Changsha: National University of defense technology,
2015.

[2] 安永泉, 李晋华, 王志斌, 等. 基于大气氧光谱吸收特性的单目单波 段被动测距[J]. 物理学报, 2013, 62(14): 1442101-1442107.

AN Yongquan, LI Jinhua, WANG Zhibin, et al. Mono-station and single-band passive ranging based on oxygen spectrum[J]. Acta Phys. Sin., 2013, 62(14): 1442101-1442107.

[3] Dowski E, Cathey T. Single-lens single-image incoherent passive-ranging systems[J]. Applied Optics, 1994, 33(29): 6762-6773.

[4] HUANG S, LIN T, ZHANG T. A modified method of passive ranging using optical flow of target infrared images[C]/Proc. of SPIE, 2005, 6044: 60441N.

[5] 付小宁, 陈立强, 董怘. 基于改进 Elman 神经网络的红外被动测距算 法研究[J]. 红外技术, 2019, 41(6): 540-544.

FU Xiaoning, CHEN Liqiang, DENG Que. Passive Ranging Algorithm Based on Improved Elman Neural Network[J]. Infrared Technology, 2019, 41(6): 540-544.

[6] 安永泉, 王志斌, 李晋华, 等. 基于 $\mathrm{O}_{2}$ 吸收特性单站被动测距机理研 究 [J]. 红外与激光工程, 2015, 44(1): 310-316.

AN Yongquan, WANG Zhibin, LI Jinhua, et al. Research on single station passive ranging based on $\mathrm{O}_{2}$ absorption characteristics[J]. Infrared and Laser Engineering, 2015, 44(1): 310-316.

[7] Anderson J, Hawks M, Gross K, et al. Flight test of an imaging $\mathrm{O}_{2}(\mathrm{X}-\mathrm{b})$ monocular passive ranging instrument[C]/Proc. of SPIE, 2011, 8020: 8020051-80200512.

[8] Diedenoven B, Hasekamp P, Aben I. Surface pressure retrieval from SCIAMACHY measurements in the $\mathrm{O}_{2}$ A Band: validation of the measurements and sensitivity on aerosols[J]. Atmospheric Chemistry And Physics, 2005, 5(8): 2109-2120.

[9] 宗鹏飞, 王志斌, 陈媛媛, 等. 基于氧气 A 吸收带的 baseline 拟合距离 反演算法 [J]. 光散射学报, 2013, 25(1): 79-84. ZONG Pengfei, WANG Zhibin, CHEN Yuanyuan, et al. Oxygen 'A' absorption band LBLRTM and distance inversion algorithm based on basedline fitting[J]. The Journal of Light Scattering, 2013, 25(1): 79-84.

[10] 张瑜, 刘秉琦, 间宗群, 等. 背景辐射对被动测距精度影响分析及实 验研究[J]. 物理学报, 2015, 64(3): 342161-342166.

ZHANG Yu, LIU Bingqi, YAN Zongqun, et al. Influence of background radiation on the precision of passive ranging[J]. Acta. Phys. Sin., 2015, 64(3): 342161-342166.

[11] YAN Z, LIU B, HUA W, et al. Theoretical analysis of the effect of meteorologic factors on passive ranging technology based on oxygen absorption spectrum[J]. Optik, 2013, 124(23): 6450-6455. 